\title{
Neurophysiological mechanisms of exertional dyspnoea in fibrotic interstitial lung disease
}

\author{
Michele R. Schaeffer ${ }^{1,2}$, Christopher J. Ryerson ${ }^{1,3}$, Andrew H. Ramsook (1) ${ }^{1,2}$, \\ Yannick Molgat-Seon ${ }^{1,4}$, Sabrina S. Wilkie ${ }^{1}$, Satvir S. Dhillon ${ }^{1}$, \\ Reid A. Mitchell ${ }^{1,2}$, A. William Sheel ${ }^{4}$, Nasreen Khalil ${ }^{3}$, Pat G. Camp ${ }^{1,2}$ and \\ Jordan A. Guenette 1,2,4 $^{1,4}$
}

Affiliations: ${ }^{1}$ Centre for Heart Lung Innovation, Providence Health Care Research Institute, University of British Columbia, St Paul's Hospital, Vancouver, BC, Canada. ${ }^{2}$ Dept of Physical Therapy, University of British Columbia, Vancouver, BC, Canada. ${ }^{3}$ Dept of Medicine, University of British Columbia, Vancouver, BC, Canada. ${ }^{4}$ School of Kinesiology, University of British Columbia, Vancouver, BC, Canada.

Correspondence: Jordan A. Guenette, Centre for Heart Lung Innovation, 166-1081 Burrard Street, Vancouver, BC, Canada, V6Z 1Y6. E-mail: jordan.guenetteahli.ubc.ca

@ERSpublications

Neural respiratory drive is a strong independent predictor of dyspnoea in patients with fibrotic ILD http://ow.ly/MXjd30hcabH

Cite this article as: Schaeffer MR, Ryerson CJ, Ramsook $\mathrm{AH}$, et al. Neurophysiological mechanisms of exertional dyspnoea in fibrotic interstitial lung disease. Eur Respir J 2018; 51: 1701726 [https://doi.org/ 10.1183/13993003.01726-2017].

ABSTRACT Our understanding of the mechanisms of dyspnoea in fibrotic interstitial lung disease (ILD) is incomplete. The aims of this study were two-fold: 1) to determine whether dyspnoea intensity is better predicted by neural respiratory drive $(\mathrm{NRD})$ or neuromechanical uncoupling (NMU) of the respiratory system in fibrotic ILD, and 2) to examine the effect of breathing $60 \%$ oxygen on NRD, NMU and dyspnoea ratings.

Fourteen patients with fibrotic ILD were included. Visit 1 comprised a familiarisation incremental cycle exercise test, Visit 2 comprised a normoxic incremental cycling test to address Aim 1, and Visits 3 and 4 consisted of constant-load cycling while breathing room air or $60 \%$ oxygen to address Aim 2 . Diaphragmatic electromyography (EMGdi) was used as a surrogate of NRD. NMU was calculated as the ratio between EMGdi (\%max) and tidal volume (\%vital capacity).

On adjusted analysis, NMU and its constituents were all significantly associated with dyspnoea ratings during incremental cycling, with EMGdi having the strongest correlation. The between-treatment change in dyspnoea ratings during constant load cycling was only correlated with change in exercise endurance time and NMU.

Dyspnoea more strongly reflected the level of EMGdi than NMU in fibrotic ILD. However, the improvement in dyspnoea with $60 \%$ oxygen was better predicted by improvements in NMU. 


\section{Introduction}

Dyspnoea is a common and debilitating symptom in patients with fibrotic interstitial lung disease (ILD). In this population, dyspnoea is an independent predictor of morbidity and mortality, and is associated with decreased health-related quality of life and reductions in functional capacity $[1,2]$. Despite its clinical importance, our understanding of the mechanisms of dyspnoea in fibrotic ILD remains incomplete.

The mechanisms of dyspnoea, in both health and disease, are complex and multifactorial [3]. However, accumulating evidence suggests that neural respiratory drive (NRD) and the imbalance between NRD and the mechanical response of the respiratory system (i.e. neuromechanical uncoupling (NMU)) are key contributors [4]. A strong relationship has recently been established between NRD, estimated using crural diaphragm electromyography (EMGdi), and dyspnoea intensity ratings in patients with ILD [5]. Furthermore, a study in chronic obstructive pulmonary disease (COPD) showed that dyspnoea intensity was strongly associated with both NRD and NMU; however, the correlation between dyspnoea intensity and NRD was more significant [6]. It is unknown whether these findings are also translatable to patients with ILD.

Treatment options to reduce dyspnoea in ILD are limited; however, recent evidence suggests that breathing a hyperoxic inspirate (inspiratory oxygen fraction $\left(\mathrm{FIO}_{2}\right)=0.60$ ) significantly reduces dyspnoea intensity ratings and can eliminate or delay the onset of 'unsatisfied inspiration' during exercise [7]. To our knowledge, no study has investigated the effect of supplemental oxygen on NRD and/or NMU in ILD in order to determine which outcome is more strongly associated with reduced exertional dyspnoea ratings. Accordingly, the aims of this study were to determine whether dyspnoea intensity in fibrotic ILD is better predicted by NRD or NMU (Aim 1), and to examine the effect of $60 \%$ oxygen on EMGdi-derived measures of NRD and NMU (Aim 2).

\section{Methods}

\section{Participants}

This study included 14 fibrotic ILD patients with isolated lung involvement that simultaneously participated in a previously published study $(n=20)$ examining the effects of hyperoxia on cycle endurance time and dyspnoea [7]. All patients had resting arterial oxygen saturation $\geqslant 92 \%$. Patients who had recently completed pulmonary rehabilitation ( $\leqslant 6$ weeks prior to enrolment), or with significant emphysema, pulmonary hypertension, extrapulmonary disease and/or other contraindications to exercise were excluded from participating.

\section{Study design}

This study was a component of a larger randomised, single-blind, placebo-controlled cross-over study (ClinicalTrials.gov: NCT01781793) with institutional ethical approval (H13-00059). Written informed consent was obtained from all patients prior to enrolment. Patients visited the laboratory on four occasions separated by $\geqslant 48 \mathrm{~h}$ between visits. Medical history, chronic activity-related dyspnoea questionnaires, pulmonary function testing, and a symptom-limited incremental cycle exercise test with dyspnoea assessment for familiarisation purposes were completed at Visit 1. During Visit 2, patients performed pulmonary function testing and a symptom-limited incremental cycle exercise test while equipped with an EMGdi catheter. Visit 2 data were used to address the primary aim. During Visits 3 and 4, patients performed pulmonary function testing and a symptom-limited constant-load cycle exercise test while breathing room air $\left(\mathrm{FiO}_{2}=0.21\right)$ or hyperoxia $\left(\mathrm{FiO}_{2}=0.60\right)$, in randomised order. Gases were delivered into a non-diffusing Douglas bag connected to a two-way non-rebreathing valve via large-bore tubing (Hans Rudolph, Inc., Shawnee, KS, USA). The gas delivery system was identical for both conditions, and patients were blinded to the inspired gas condition. Data from Visits 3 and 4 were used to address the secondary aim of the study.

Support statement: This study was funded by the British Columbia Lung Association (BCLA), an Emerging Research Leaders Initiative Grant from the Canadian Respiratory Research Network, and seed funding from the Dept of Physical Therapy at the University of British Columbia (Vancouver, BC, Canada). M.R. Schaeffer was supported by fellowships from the University of British Columbia and BCLA. C.J. Ryerson and P.G. Camp were supported by Scholar Awards from the Michael Smith Foundation for Health Research (MSFHR). A.H. Ramsook was supported by the University of British Columbia 4-Year Fellowship (UBC 4YF) and an Alexander Graham Bell Canada Graduate Scholarship from the Natural Sciences and Engineering Research Council of Canada (NSERC). Y. Molgat-Seon was supported by the UBC 4YF and a Postgraduate Scholarship from the NSERC. J.A. Guenette was supported by a Scholar Award from the MSFHR, a Clinical Rehabilitation New Investigator Award from the Canadian Institutes of Health Research, and a New Investigator Award from the Providence Health Care Research Institute and St Paul's Hospital Foundation. The funders had no role in the study design, data collection and analysis, or preparation of the manuscript. Funding information for this article has been deposited with the Crossref Funder Registry.

Conflict of interest: Disclosures can be found alongside this article at erj.ersjournals.com 


\section{Pulmonary function}

Patients performed spirometry, plethysmography, 12-s maximal voluntary ventilation, and single-breath diffusing capacity of the lungs for carbon monoxide (DLCO), according to established guidelines [8-10]. Values were measured using a commercially available system (Vmax Encore 229, V62J Autobox; CareFusion, Yorba Linda, CA, USA) and expressed as a percentage of predicted values $[11,12]$.

\section{Exercise testing protocol}

Exercise was conducted using an electronically braked cycle ergometer (Ergoselect 200P; Ergoline, Bitz, Germany) at a self-selected cadence $\geqslant 60$ revolutions per minute. All tests started with 6 min of rest followed by a 1-min warm-up of unloaded pedalling. Incremental tests proceeded with $15-\mathrm{W}$ stepwise increases in work-rate, starting at $15 \mathrm{~W}$, every 2 min until symptom limitation. Peak work-rate was defined as the highest work-rate sustained for at least $30 \mathrm{~s}$. Constant-load exercise tests proceeded with an immediate increase in work-rate to $75 \%$ of the peak incremental work-rate, which was sustained until symptom limitation. Iso-time was defined as the highest equivalent submaximal time achieved on both constant-load exercise tests performed by a given patient under both conditions. No verbal encouragement was provided during constant-load exercise tests.

\section{Symptom evaluation}

Patients rated the intensity of 'breathing discomfort' (dyspnoea) and 'leg discomfort' at rest, every 2 min during exercise, and at peak exercise using the modified Borg 0-10 category-ratio scale [13]. The scale's end-points were anchored such that 0 represented 'no breathing/leg discomfort' and 10 represented 'the most severe breathing/leg discomfort ever experienced or imagined'.

\section{Cardiorespiratory responses to exercise}

Standard cardiorespiratory responses were measured on a breath-by-breath basis at rest and during exercise (Vmax Encore 229; CareFusion). This metabolic cart has not been validated with an $\mathrm{FIO}_{2}$ of 0.60 ; therefore, differences in gas exchange parameters (i.e. oxygen consumption and carbon dioxide production) and gas exchange-derived parameters (i.e. ventilatory equivalents and the respiratory exchange ratio) were not compared between conditions (Visits 3 and 4). Flow was calibrated using a 3-L calibration syringe connected to the breathing apparatus and using the appropriate gas mixture from the Douglas bag at a wide range of flow rates. The inflection point in tidal volume relative to minute ventilation $\left(V_{\mathrm{T}} / V^{\prime} \mathrm{E}\right)$ was determined for each patient by examining 30-s averaged data throughout incremental exercise (Visit 2). The time at which the corresponding VT was achieved during Visits 3 and 4, if at all, was also identified using the 30-s averaged data from those visits. Arterial oxygen saturation was estimated via finger pulse oximetry (Radical-7 Pulse CO Oximeter; Masimo Corp., Irvine, CA, USA). Heart rate was recorded using 12-lead electrocardiography.

\section{Diaphragm electromyography}

Crural diaphragm electromyography was measured using a multi-pair oesophageal electrode catheter (Guangzhou Respiratory Institute Medical Equipment and Scientific Co. Ltd, Guangzhou, China) and was used as a surrogate of NRD [14]. Diaphragm electromyography was collected, processed and analysed as previously described [15]. Briefly, the catheter consists of five consecutive recording pairs, and this was passed through the nose and positioned based on the amplitude of EMGdi signals obtained simultaneously from these recording pairs during tidal breathing. To minimise discomfort, a lidocaine hydrochloride non-aerosol spray (Lidodan; Odan Laboratories, Montreal, Canada) was administered in the nasal and pharyngeal passages. Raw EMGdi signals were sampled at $2 \mathrm{kHz}$ using a PowerLab 16/35 analogue-to-digital converter (ADInstruments, Castle Hill, Australia), processed with a $60-\mathrm{Hz}$ notch filter (Model 08-GL biological Amplifier, Guangzhou Respiratory Institute Medical Equipment and Scientific Co. Ltd) as well as a band-pass filter between $20 \mathrm{~Hz}$ and $1 \mathrm{kHz}$ using LabChart Pro Version 7.3.7 software (ADInstruments), and converted to a root mean square (RMS) using a time constant of $0.1 \mathrm{~s}$. The maximum RMS value for each inspired breath was manually selected between QRS complexes to avoid the influence of cardiac artefact on the EMG signal. The electrode pair with the largest amplitude for a given breath was used for the analysis. Data were reported as a percentage of maximal EMGdi, which was defined as the highest RMS value recorded during an inspiratory capacity manoeuvre at rest or during exercise. We used inspiratory capacity manoeuvres exclusively instead of using multiple maximal inspiratory manoeuvres (e.g. sniffs and maximal inspiratory pressure manoeuvres) to obtain maximal EMGdi. This was done to standardise the normalisation procedure across all subjects, and because our previous work shows that inspiratory capacity manoeuvres always result in the greatest EMGdi activity [16]. NMU was defined as the ratio of EMGdi expressed as a percentage of maximum to $V \mathrm{~T}$ expressed as a percentage of vital capacity (VC) (i.e. EMGdi (\%max):VT (\%VC)). 


\section{Statistical analyses}

Exercise variables were measured continuously, with mean values determined over a 30-s epoch linked to the time of dyspnoea assessment. We determined the association of dyspnoea intensity ratings with NMU and its individual components (i.e. EMGdi (\%max) and $V \mathrm{~T}$ (\%VC)), using an unadjusted repeated-measures linear regression. All of these predictors were then applied to a multivariate model, also using repeated-measures analysis, to determine independent predictors. Variables included in the multivariate model were assessed for multicollinearity. Paired t-tests were used to compare outcomes between the room air and hyperoxic conditions at iso-time and peak exercise. Spearman's correlation coefficients were used to make intra-subject, between-treatment comparisons of dyspnoea intensity ratings with select physiological variables during constant-load exercise at iso-time. Data are presented as mean $\pm \mathrm{SD}$ or median (interquartile range) where appropriate. Statistical significance was set at $\mathrm{p}<0.05$. Data were analysed using Stata 11.2 software (StataCorp LP, College Station, TX, USA).

\section{Results}

\section{Participants}

Patient characteristics and peak exercise data are summarised in table 1. On average, patients had moderate physiological impairment, as demonstrated by reduced forced vital capacity and diffusing capacity of the lungs for carbon monoxide, and reduced functional capacity, as evidenced by reduced peak aerobic capacity and work-rate relative to predicted values $[11,12,17]$. The $V \mathrm{~T} / V^{\prime} \mathrm{E}$ inflection point occurred at $76 \pm 28 \mathrm{~W}$ during incremental exercise. None of the patients qualified for supplemental oxygen, at rest or with ambulation, according to regional criteria [18]. No cardiac abnormalities were noted during exercise testing.

\section{Associations with dyspnoea}

During the incremental cycle exercise test on Visit 2, 97 observations were obtained. One patient was excluded from this analysis (Aim 1) owing to lack of EMGdi data on Visit 2; the patient refused the catheter. Dyspnoea intensity ratings were significantly associated with NMU, EMGdi (\%max) and $V \mathrm{~T}$ (\% VC) (all p<0.001, fig. 1). With adjusted analysis, EMGdi (\%max), NMU and VT (\%VC) remained significantly associated with dyspnoea intensity ratings $(\mathrm{p}<0.001, \mathrm{p}=0.006$ and $\mathrm{p}=0.02$, respectively), with EMGdi having the strongest correlation (fig. 1).

\section{Effects of hyperoxia}

The sensory and physiological effects of hyperoxia during constant-load cycle exercise are summarised in table 2. Three patients were excluded from this analysis (Aim 2); these patients refused the catheter for at least one of these visits and/or did not complete both visits. The average time between constant-load cycle exercise tests was $6 \pm 2$ days. The mean work-rate for the constant-load cycle exercise tests was $71 \pm 27 \mathrm{~W}$, and the median iso-time was $6.0(4.0-10.0) \mathrm{min}$. Constant-load cycle exercise endurance time increased significantly with hyperoxia compared to that with room air $(24.2 \pm 11.1$ versus $9.6 \pm 8.0 \mathrm{~min}$, respectively; $\mathrm{p}<0.001)$.

Dyspnoea intensity ratings were similar between room air and hyperoxia at peak exercise $(\mathrm{p}=0.38)$, but significantly reduced at iso-time with hyperoxia versus room air $(1.8 \pm 1.9$ versus $3.8 \pm 3.0$ Borg $0-10$ units, respectively; $\mathrm{p}=0.005$; table 2). EMGdi was significantly reduced during constant-load cycle exercise at iso-time $(p<0.001)$ and peak exercise $(p=0.005)$ with hyperoxia versus room air (table 2$)$. NMU was significantly improved at iso-time with hyperoxia versus room air $(p=0.003$; table 2). The between-treatment change in iso-time dyspnoea ratings during constant-load cycle exercise was only correlated with the change in exercise endurance time $(\mathrm{r}=-0.67, \mathrm{p}=0.02)$ and NMU $(\mathrm{r}=0.63, \mathrm{p}=0.04)$. Additional Spearman's correlation coefficients are shown in table 3.

During constant-load cycle exercise with room air, all 11 patients included in the analysis for Aim 2 achieved or surpassed a $V \mathrm{~T}$ that corresponded to the $V \mathrm{~T} / V^{\prime} \mathrm{E}$ inflection point identified on the incremental test. Two of these patients did not achieve this $V \mathrm{~T}$ with hyperoxia, and the time at which this VT was achieved was delayed with hyperoxia versus room air in the remaining nine patients $(5.5 \pm 5.1$ versus $1.9 \pm 0.7 \mathrm{~min}$, respectively; $\mathrm{p}=0.06$ ). Measures of dyspnoea, EMGdi (\%max) and NMU were not different at this measurement time between treatments $(p=0.12, p=0.12$ and $p=0.09$, respectively).

\section{Discussion}

We sought to determine whether dyspnoea intensity in fibrotic ILD is primarily determined by NRD or NMU, and to examine the impact of $60 \%$ oxygen on NRD and NMU. Our main findings were as follows: 1) dyspnoea intensity ratings were significantly correlated with both EMGdi and NMU during incremental cycle exercise, but with a stronger correlation between dyspnoea and EMGdi; and 2) the improvements in 


\section{TABLE 1 Patient characteristics and maximal exercise data}

\begin{tabular}{|c|c|}
\hline Descriptive characteristic & Value \\
\hline Age years & $66 \pm 10$ \\
\hline Male:female ratio & $10: 4$ \\
\hline $\mathrm{BMI} \mathrm{kg} \cdot \mathrm{m}^{-2}$ & $29 \pm 5$ \\
\hline \multicolumn{2}{|l|}{ Diagnosis n } \\
\hline IPF & 7 \\
\hline $\mathrm{HP}$ & 2 \\
\hline Idiopathic LIP & 1 \\
\hline Polymyositis-associated ILD"\# & 1 \\
\hline Scleroderma-associated ILD" & 1 \\
\hline Unclassifiable ILD & 1 \\
\hline Undifferentiated CTD & 1 \\
\hline Time since diagnosis months & $40.1(31.1-64.3)$ \\
\hline Surgical lung biopsy $n$ & 7 \\
\hline Modified MRC Dyspnoea 0-4 scale & $1.5 \pm 1.2$ \\
\hline Oxygen cost diagram mm & $62 \pm 17$ \\
\hline \multicolumn{2}{|l|}{ Pulmonary function } \\
\hline FEV $1 \%$ pred & $77 \pm 15$ \\
\hline FVC \% pred & $71 \pm 16$ \\
\hline $\mathrm{FEV}_{1} / \mathrm{FVC} \%$ & $80 \pm 8$ \\
\hline TLC \% pred & $64 \pm 11$ \\
\hline DLco \% pred & $45 \pm 15$ \\
\hline \multicolumn{2}{|l|}{ Peak incremental exercise } \\
\hline Dyspnoea 0-10 Borg scale & $5.7 \pm 2.3$ \\
\hline Leg discomfort 0-10 Borg scale & $5.6 \pm 2.1$ \\
\hline$V^{\prime} \mathrm{O}_{2} \mathrm{~mL} \cdot \mathrm{kg}^{-1} \cdot \mathrm{min}^{-1}$ & $21.1 \pm 6.2$ \\
\hline$V^{\prime} \mathrm{O}_{2} \%$ pred & $70 \pm 23$ \\
\hline RER & $1.08 \pm 0.08$ \\
\hline Work rate $\mathrm{W}$ & $93 \pm 39$ \\
\hline Work rate $\%$ pred & $59 \pm 17$ \\
\hline Heart rate bpm & $132 \pm 20$ \\
\hline Heart rate $\%$ pred & $87 \pm 10$ \\
\hline$V^{\prime} \mathrm{E} L \cdot \mathrm{min}^{-1}$ & $76 \pm 20$ \\
\hline$V^{\prime} E / M V V \%$ & $72 \pm 23$ \\
\hline $\mathrm{SpO}_{2} \%$ & $90 \pm 5$ \\
\hline EMGdi \%max & $69 \pm 15$ \\
\hline NMU EMGdi (\%max):VT (\% VC) & $1.4 \pm 0.4$ \\
\hline
\end{tabular}

Data are presented as mean \pm SD or median (interquartile range), unless otherwise stated. BMI: body mass index; IPF: idiopathic pulmonary fibrosis; HP: hypersensitivity pneumonitis; LIP: lymphoid interstitial pneumonia; ILD: interstitial lung disease; CTD: connective tissue disease; MRC: Medical Research Council; FEV1: forced expiratory volume in $1 \mathrm{~s}$; FVC: forced vital capacity; TLC: total lung capacity; DLCo: diffusing capacity of the lungs for carbon monoxide; $V^{\prime} \mathrm{O}_{2}$ : oxygen consumption; RER: respiratory exchange ratio; bpm: beats per minute; $V^{\prime} \mathrm{E}$ : minute ventilation; $\mathrm{MVV}$ : maximal voluntary ventilation; $\mathrm{SpO}_{2}$ : arterial oxygen saturation measured by pulse oximetry; EMGdi: electromyogram of the crural diaphragm; NMU: neuromechanical uncoupling; $V_{T}$ : tidal volume; VC: vital capacity. "\#: these patients had no extrapulmonary manifestations.

dyspnoea intensity ratings at iso-time during constant-load cycle exercise with hyperoxia were significantly correlated with the change in NMU, but not with the change in EMGdi.

It has been proposed that increased dyspnoea is a reflection of increased levels of NRD in both health and disease [4]. There is also evidence that central and peripheral afferent feedback pathways may neuromodulate the intensity of perceived dyspnoea through direct effects on NRD [4]. Accordingly, increased dyspnoea intensity ratings in patients with cardiopulmonary disease likely correspond to the awareness of an increased NRD needed to meet an increased ventilatory demand in the context of increased mechanical loading and/or reduced capacity of the respiratory muscles. For example, patients with ILD demonstrate reduced compliance and increased static recoil pressure of the lung due to fibrosis. The static pressure-volume curve of the lungs is therefore shifted downwards and to the right $[19,20]$. The pressure-volume relationship of the entire respiratory system is therefore contracted along its volume axis [21], whereby resting inspiratory capacity and inspiratory reserve volume are generally diminished. This 

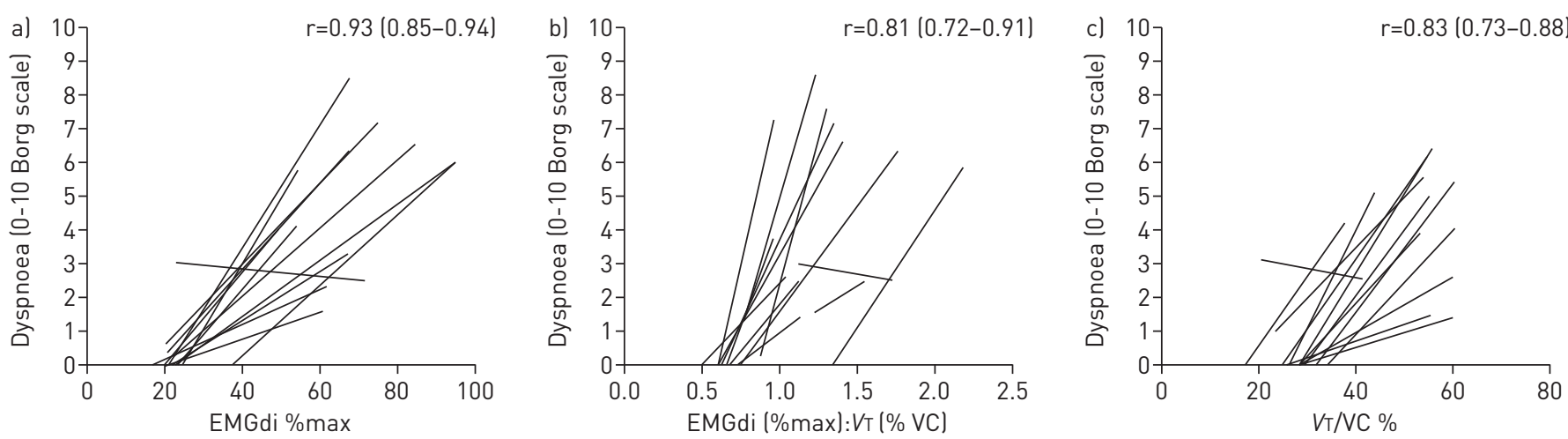

FIGURE 1 Trend lines for individual patients demonstrating the relationship between dyspnoea intensity and a) electromyography of the diaphragm (EMGdi), b) neuromechanical uncoupling, and c) tidal volume ( $\mathrm{V}$ ) expressed as a percentage of vital capacity (VC). Reported r-values are expressed as median (interquartile range) from Spearman's correlations within each patient.

reduction in baseline reserve translates to less potential for $V$ T expansion during exercise [22, 23]. The ILD patients in the present study achieved critical constraints on $V \mathrm{~T}$ expansion at markedly lower absolute work-rates than what has been previously documented in healthy men and women of similar ages [24]. Thus, ILD patients are likely to have increased elastic loading of the respiratory muscles [21], which necessitates greater respiratory muscle force for inspiration [25], and therefore an increased NRD [5], in order to meet ventilatory requirements.

FAISAL et al. [5] showed that the relationship between EMGdi and dyspnoea intensity is preserved in patients with ILD and COPD, as well as healthy controls. This supports the hypothesis that NRD is an important component of the physiological basis of increased dyspnoea intensity, as disease-specific differences in respiratory function and/or capacity, and therefore afferent feedback pathways, do not influence this association [4]. Indeed, another recent study in COPD patients [6] examined the relationships of dyspnoea intensity with NRD and NMU, concluding that dyspnoea intensity is closely related to EMGdi and NMU, but that the relationship between dyspnoea intensity ratings and EMGdi was comparatively stronger. This is consistent with our findings, suggesting that increased NRD largely contributes to increased dyspnoea intensity in patients with fibrotic ILD, at least during normoxic incremental exercise.

TABLE 2 Selected sensory and physiological parameters at rest, iso-time and peak exercise for constant-load cycle exercise tests with room air and hyperoxia

\begin{tabular}{|c|c|c|c|c|}
\hline & \multicolumn{2}{|c|}{ Iso-time } & \multicolumn{2}{|c|}{ Peak } \\
\hline & Room air & Hyperoxia & Room air & Hyperoxia \\
\hline Dyspnoea 0-10 Borg scale & $3.8 \pm 3.0$ & $1.8 \pm 1.9 * *$ & $5.0 \pm 2.7$ & $4.5 \pm 3.7$ \\
\hline Leg discomfort 0-10 Borg scale & $3.4 \pm 2.1$ & $2.1 \pm 1.5$ & $4.7 \pm 1.8$ & $5.9 \pm 2.2$ \\
\hline $\mathrm{SpO}_{2} \%$ & $89 \pm 3$ & $98 \pm 2 * * *$ & $88 \pm 4$ & $98 \pm 1 * * *$ \\
\hline Heart rate bpm & $125 \pm 22$ & $114 \pm 19 * * *$ & $127 \pm 21$ & $125 \pm 22$ \\
\hline Heart rate $\%$ pred & $83 \pm 14$ & $76 \pm 13^{* * *}$ & $85 \pm 13$ & $83 \pm 15$ \\
\hline$V^{\prime} \mathrm{E} L \cdot \mathrm{min}^{-1}$ & $74 \pm 18$ & $53 \pm 14^{* * *}$ & $76 \pm 20$ & $62 \pm 13^{* *}$ \\
\hline$V^{\prime}$ E/MVV \% & $71 \pm 26$ & $50 \pm 18 * * *$ & $73 \pm 26$ & $59 \pm 20 * *$ \\
\hline$V_{T} \mathrm{~L}$ & $1.58 \pm 0.48$ & $1.39 \pm 0.44^{* * *}$ & $1.50 \pm 0.46$ & $1.29 \pm 0.36^{* *}$ \\
\hline$F B$ breaths $\cdot \min ^{-1}$ & $49 \pm 15$ & $40 \pm 12^{* *}$ & $54 \pm 19$ & $50 \pm 15$ \\
\hline EELV \% TLC & $55 \pm 5$ & $56 \pm 7$ & $54 \pm 7$ & $57 \pm 7$ \\
\hline EILV \% TLC & $93 \pm 5$ & $89 \pm 5^{*}$ & $91 \pm 6$ & $88 \pm 6^{*}$ \\
\hline $\mathrm{PETCO}_{2} \mathrm{mmHg}$ & $33 \pm 6$ & $39 \pm 6 * * *$ & $32 \pm 7$ & $37 \pm 7^{* * *}$ \\
\hline EMGdi \%max & $67 \pm 16$ & $49 \pm 12 * * *$ & $68 \pm 18$ & $53 \pm 13^{* *}$ \\
\hline NMU EMGdi (\%max):V个 $(\% \mathrm{VC})$ & $1.3 \pm 0.3$ & $1.0 \pm 0.2^{* *}$ & $1.3 \pm 0.4$ & $1.2 \pm 0.2$ \\
\hline
\end{tabular}

Data are presented as mean $\pm \mathrm{SD}$. $\mathrm{SpO}_{2}$ : arterial oxygen saturation measured by pulse oximetry; bpm: beats per minute; $V^{\prime} \mathrm{E}$ : minute ventilation; MVV: maximal voluntary ventilation; $V_{\mathrm{T}}$ : tidal volume; $F_{\mathrm{B}}$ : breathing frequency; EELV: end-expiratory lung volume; TLC: total lung capacity; EILV: end-inspiratory lung volume; $\mathrm{PETCO}_{2}$ : end-tidal carbon dioxide tension; EMGdi: electromyography of the crural diaphragm; NMU: neuromechanical uncoupling; VC: vital capacity. Significantly different from room air: ${ }^{*}: p<0.05 ;{ }^{* *}: p<0.01$; $* * *: p<0.001$. 
TABLE 3 Spearman's correlations for selected parameters with the change in dyspnoea intensity ratings at iso-time with hyperoxia versus room air

\begin{tabular}{|c|c|c|}
\hline & r-value & p-value \\
\hline$\Delta \mathrm{EET} \min$ & -0.67 & 0.02 \\
\hline FVC $\%$ pred & -0.43 & 0.18 \\
\hline DLco \% pred & -0.11 & 0.75 \\
\hline Peak incremental $V^{\prime} \mathrm{O}_{2} \%$ pred & 0.11 & 0.75 \\
\hline$\Delta \mathrm{SpO}_{2}$ at iso-time $\%$ & -0.04 & 0.91 \\
\hline$\Delta \mathrm{SpO}_{2}$ at peak exercise $\%$ & -0.06 & 0.86 \\
\hline$\Delta V^{\prime} \mathrm{E}$ at iso-time $\mathrm{L} \cdot \mathrm{min}^{-1}$ & 0.14 & 0.68 \\
\hline$\Delta V_{\mathrm{T}}$ at iso-time $\mathrm{L}$ & -0.46 & 0.16 \\
\hline$\Delta F_{B}$ at iso-time breaths $\cdot \min ^{-1}$ & 0.23 & 0.50 \\
\hline$\Delta \mathrm{EELV}$ at iso-time $\% \mathrm{TLC}$ & 0.37 & 0.26 \\
\hline$\Delta$ EILV at iso-time $\%$ TLC & 0.27 & 0.43 \\
\hline$\Delta$ EMGdi at iso-time $\% \max$ & 0.41 & 0.21 \\
\hline$\Delta \mathrm{NMU}$ at iso-time EMGdi (\%max): $V_{\mathrm{T}}(\% \mathrm{VC})$ & 0.63 & 0.04 \\
\hline
\end{tabular}

$\Delta$ : difference between selected parameter with hyperoxia versus room air; EET: exercise endurance time; FVC: forced vital capacity; $D\left\llcorner C 0\right.$ : diffusing capacity of the lungs for carbon monoxide; $V^{\prime} \mathrm{O}_{2}$ : oxygen consumption; $\mathrm{SpO}_{2}$ : arterial oxygen saturation measured by pulse oximetry; $V^{\prime} \mathrm{E}$ : minute ventilation; $V_{\mathrm{T}}$ : tidal volume; FB: breathing frequency; EELV: end-expiratory lung volume; TLC: total lung capacity; EILV: end-inspiratory lung volume; EMGdi: electromyography of the crural diaphragm; NMU: neuromechanical uncoupling; VC: vital capacity.

We recently demonstrated significant reductions in iso-time dyspnoea intensity ratings during constant-load exercise with hyperoxia versus room air [7]. However, no signification correlations were found between the reduction in dyspnoea intensity ratings and the between-treatment changes in standard ventilatory measures during exercise. We reasoned that a reduction in dyspnoea intensity ratings with hyperoxia may therefore be associated with improvements in neurophysiological parameters such as NRD and/or NMU. However, despite EMGdi being more significantly correlated with dyspnoea intensity ratings during normoxic incremental exercise, the improvement in dyspnoea intensity ratings with hyperoxia were only significantly correlated with an improvement in NMU and exercise performance (i.e. endurance time). This suggests that hyperoxia reduces dyspnoea via multiple mechanisms, with a combined beneficial effect on both NRD and respiratory mechanics.

Previous studies have shown that hyperoxia improves oxygen delivery to the skeletal muscles by widening the arteriovenous oxygen content difference, thereby increasing the anaerobic threshold and delaying the onset of metabolic acidosis and skeletal muscle fatigue [26, 27]. The subsequent reduction in $V^{\prime} \mathrm{E}$ at iso-time and peak exercise in the present study may have reflected, at least in part, both a reduction in EMGdi as well as $V \mathrm{~T}$, with attendant improvements in perceived breathlessness. This is further supported by the delayed onset of the $V \mathrm{~T} / V^{\prime} \mathrm{E}$ inflection with hyperoxia versus room air. NMU was defined in this study as the ratio of EMGdi (\%max) to $V \mathrm{~T}(\% \mathrm{VC})$, and was therefore a direct representation of the interaction between EMGdi and VT. In this context, had only EMGdi been reduced and VT held constant with hyperoxia, NMU would have decreased (i.e. improved). Alternatively, had only $V \mathrm{~T}$ been reduced and EMGdi held constant with hyperoxia, NMU would have increased (i.e. worsened). We observed reductions in both EMGdi and $V \mathrm{~T}$ with hyperoxia versus room air, with a relatively larger reduction in EMGdi (26 \pm 11 versus $12 \pm 6 \%$, respectively). Accordingly, our study suggests that hyperoxia reduces dyspnoea mainly via an improvement in NMU, which reflects an optimised relationship between EMGdi and VT. The interaction between the improvement in NRD and the change in VT seems to have a more important effect than the improvement in NRD alone.

\section{Limitations}

There are some limitations to this study that must be acknowledged. First, we used EMGdi as a surrogate of NRD, and the limitations of this method have been well described [5]. Second, while the diaphragm is the primary inspiratory muscle, we cannot ignore the potential contribution of extra-diaphragmatic inspiratory muscles, such as the sternocleidomastoid, scalene and intercostal muscles. Changes in the ventilatory response with hyperoxia could elicit changes in respiratory muscle recruitment patterns and their relative contributions, which could influence measures of NMU. Third, owing to safety reasons, it was not feasible to blind all study personnel to the study condition. However, we do not believe this impacted our results as all test procedures were rigorously standardised, including an absence of verbal 
encouragement for all constant-load exercise tests. Fourth, we acknowledge that this invasive study had a small sample size, and therefore may not be representative of all patients with fibrotic ILD. Finally, we included a variety of ILD subtypes; however, all patients had isolated lung involvement. Owing to the similar physiology of these diseases, we expect the physiological findings to be similar across these subtypes.

\section{Conclusion}

Our data suggest that the intensity of dyspnoea more likely reflects increased NRD, as estimated by EMGdi, rather than NMU, in patients with ILD during normoxic incremental exercise. However, attenuation of dyspnoea intensity ratings with hyperoxia was correlated with improvements in NMU, but not EMGdi. This suggests that the combined benefits of hyperoxia on the coupling between NRD and the mechanical output of the respiratory system are more important in relieving dyspnoea than the effects on NRD alone. Future research is needed to better isolate the independent effects of EMGdi and the mechanical output of the respiratory system (i.e. VT) on dyspnoea during exercise in patients with ILD. This knowledge may aid in the development of symptom-based treatment options in this population.

\section{Acknowledgements}

The authors would like to thank all of the patients for their enthusiastic participation in this study. All authors played a role in the content and writing of the manuscript. M.R. Schaeffer, C.J. Ryerson, A.W. Sheel, N. Khalil, P.G. Camp and J.A. Guenette had input into the study design and conduct of the study; M.R. Schaeffer, C.J. Ryerson, A.H. Ramsook, Y. Molgat-Seon, S.S. Wilkie, R.A. Mitchell and S.S. Dhillon collected the data; and M.R. Schaeffer, C.J. Ryerson, A.H. Ramsook, S.S. Dhillon, R.A. Mitchell and J.A. Guenette performed data analysis.

\section{References}

1 King TE, Schwarz MI, Brown K, et al. Idiopathic pulmonary fibrosis: relationship between histopathologic features and mortality. Am J Respir Crit Care Med 2001; 164: 1025-1032.

2 Swigris JJ, Kuschner WG, Jacobs SS, et al. Health-related quality of life in patients with idiopathic pulmonary fibrosis: a systematic review. Thorax 2005; 60: 588-594.

3 Parshall MB, Schwartzstein RM, Adams L, et al. An official American Thoracic Society statement: update on the mechanisms, assessment, and management of dyspnea. Am J Respir Crit Care Med 2012; 185: 435-452.

4 Jensen D, Pattinson K, Jolley C. Mechanisms of breathlessness. In: Bausewein C, Currow DC, Johnson MJ, eds. Palliative Care in Respiratory Disease (ERS Monograph). Sheffield, European Respiratory Society, 2016; pp. $111-133$.

5 Faisal A, Alghamdi BJ, Ciavaglia CE, et al. Common mechanisms of dyspnea in chronic interstitial and obstructive lung disorders. Am J Respir Crit Care Med 2016; 193: 299-309.

6 Jolley CJ, Luo YM, Steier J, et al. Neural respiratory drive and breathlessness in COPD. Eur Respir J 2015; 45: 355-364.

7 Schaeffer MR, Ryerson CJ, Ramsook AH, et al. Effects of hyperoxia on dyspnoea and exercise endurance in fibrotic interstitial lung disease. Eur Respir J 2017; 49: 1602494.

8 Miller MR, Hankinson J, Brusasco V, et al. Standardisation of spirometry. Eur Respir J 2005; 26: 319-338.

9 Wanger J, Clausen JL, Coates A, et al. Standardisation of the measurement of lung volumes. Eur Respir J 2005; 26: 511-522.

10 Macintyre N, Crapo RO, Viegi G, et al. Standardisation of the single-breath determination of carbon monoxide uptake in the lung. Eur Respir J 2005; 26: 720-735.

11 Tan WC, Bourbeau J, Hernandez P, et al. Canadian prediction equations of spirometric lung function for Caucasian adults 20 to 90 years of age: results from the Canadian Obstructive Lung Disease (COLD) study and the Lung Health Canadian Environment (LHCE) study. Can Respir J 2011; 18: 321-326.

12 Gutierrez C, Ghezzo RH, Abboud RT, et al. Reference values of pulmonary function tests for Canadian Caucasians. Can Respir J 2004; 11: 414-424.

13 Borg GA. Psychophysical bases of perceived exertion. Med Sci Sports Exerc 1982; 14: 377-381.

14 Luo YM, Moxham J, Polkey MI. Diaphragm electromyography using an oesophageal catheter: current concepts. Clin Sci 2008; 115: 233-244.

15 Schaeffer MR, Mendonca CT, Levangie MC, et al. Physiological mechanisms of sex differences in exertional dyspnoea: role of neural respiratory motor drive. Exp Physiol 2014; 99: 427-441.

16 Ramsook AH, Schaeffer MR, Syed N, et al. Which respiratory maneuvers generate the greatest electromyography activity of the inspiratory muscles? Am J Respir Crit Care Med 2015; 191: A2682.

17 Blackie SP, Fairbarn MS, McElvaney GN, et al. Prediction of maximal oxygen uptake and power during cycle ergometry in subjects older than 55 years of age. Am Rev Respir Dis 1989; 139: 1424-1429.

18 Sandberg D, Fleetham J. Home oxygen therapy in British Columbia. BC Med J 2013; 55: 149-152.

19 Yernault JC, de Jonghe $\mathrm{M}$, de Coster A, et al. Pulmonary mechanics in diffuse fibrosing alveolitis. Bull Physiopathol Respir 1975; 11: 231-244.

20 Gibson GJ, Pride NB. Pulmonary mechanics in fibrosing alveolitis: the effects of lung shrinkage. Am Rev Respir Dis 1977; 116: 637-647.

21 O’Donnell DE, Ora J, Webb KA, et al. Mechanisms of activity-related dyspnea in pulmonary diseases. Respir Physiol Neurobiol 2009; 167: 116-132.

22 Gowda K, Zintel T, McParland C, et al. Diagnostic value of maximal exercise tidal volume. Chest 1990; 98: 1351-1354.

23 Jones NL, Rebuck AS. Tidal volume during exercise in patients with diffuse fibrosing alveolitis. Bull Eur Physiopathol Respir 1979; 15: 321-328. 
24 Guenette JA, Jensen D, Webb KA, et al. Sex differences in exertional dyspnea in patients with mild COPD: physiological mechanisms. Respir Physiol Neurobiol 2011; 177: 218-227.

25 O’Donnell DE, Chau LK, Webb KA. Qualitative aspects of exertional dyspnea in patients with interstitial lung disease. J Appl Physiol 1998; 84: 2000-2009.

26 Harris-Eze AO, Sridhar G, Clemens RE, et al. Oxygen improves maximal exercise performance in interstitial lung disease. Am J Respir Crit Care Med 1994; 150: 1616-1622.

27 Powers SK, Lawler J, Dempsey JA, et al. Effects of incomplete pulmonary gas exchange on $\mathrm{VO}_{2}$ max. J Appl Physiol 1989; 66: 2491-2495. 